\title{
UNIFORM LIMITS OF SEQUENCES OF POLYNOMIALS AND THEIR DERIVATIVES
}

\author{
JOSEPH A. BALL AND THOMAS R. FANNEY
}

(Communicated by Palle E. T. Jorgensen)

\begin{abstract}
Let $E$ be a compact subset of the unit interval $[0,1]$, and let $C(E)$ denote the space of functions continuous on $E$ with the uniform norm. Consider the densely defined operator $D: C(E) \rightarrow C(E)$ given by $D p=p^{\prime}$ for all polynomials $p$. Let $\mathscr{G}$ represent the graph of $D$, that is $\mathscr{G}=\left\{\left(p, p^{\prime}\right): p\right.$ polynomials considered as a submanifold of $C(E) \times C(E)$. Write the interior of the set $E$, int $E$ as a countable union of disjoint open intervals and let $\widehat{E}$ be the union of the closure of these intervals. The main result is that the closure of $\mathscr{G}$ is equal to the set of all functions $(h, k) \in C(E) \times C(E)$ such that $h$ is absolutely continuous on $\widehat{E}$ and $k\left|\widehat{E}=h^{\prime}\right| \widehat{E}$. As a consequence, the operator $D$ is closable if and only if the set $E$ is the closure of its interior. On the other extreme, $\mathscr{G}$ is dense in $C(E) \times C(E)$ i.e. for any pair $(f, g) \in C(E) \times C(E)$, there exists a sequence of polynomials $\left\{p_{n}\right\}$ so that $p_{n} \rightarrow f$ and $p_{n}^{\prime} \rightarrow g$ uniformly on $E$, if and only if the interior int $E$ of $E$ is empty.
\end{abstract}

\section{INTRODUCTION}

The goal of this paper is to characterize those pairs of continuous functions $(f, g)$ which arise as uniform limits of polynomial pairs of the form $\left(p_{n}, p_{n}^{\prime}\right)$ (where $p_{n}^{\prime}$ is the derivative of the polynomial $p_{n}$ ) on some compact set $E \subset \mathbb{R}$. In the language of operator theory, this result amounts to a characterization of the uniform closure $\mathscr{G}^{-}$of the graph $\mathscr{G}$ of the differentiation operator $D: C(E) \rightarrow C(E)$ given by $D p=p^{\prime}$ with domain equal to the dense linear manifold of all polynomials in $C(E)$. An explicit description of the uniform closure of $\mathscr{G}$ is given by (2.1) and Theorems 2.2 and 2.9. As a corollary, a complete characterization of when $D$ is closable (i.e., when $\mathscr{G}^{-}$contains no nontrivial elements of the form $(0, k))$ is obtained in Theorem 3.1; for a discussion of this problem in the plane, see [FR]. On the other extreme, another specialization (Corollary 3.3) gives a necessary and sufficient condition for the graph of $\mathscr{G}$ to be dense in $C(E) \times C(E)$, i.e., for an arbitrary pair $(f, g)$ in $C(E) \times C(E)$ to be uniformly approximable over $E$ by a sequence of the form $\left(p_{n}, p_{n}^{\prime}\right)$, with $p_{n}$ a polynomial. Partial results on this latter problem for the case where $E$ is a compact subset of the complex plane are obtained in [B1, B2]. The general problem for the complex case where $E$ is a compact subset

Received by the editors September 20, 1990; presented at the 854th meeting of the AMS in Louisville, KY, January 1990.

1980 Mathematics Subject Classification (1985 Revision). Primary 41A10, 41A28. 
of $\mathbb{C}$ appears to be quite difficult; our purpose here is to show that a complete, explicit solution can be obtained for the case that $E$ is contained in $\mathbb{R}$ by use of elementary functional analysis.

More complicated parallel versions of these results where $C(E) \times C(E)$ is replaced by $L^{p}(\mu) \times L^{p}(\nu)$ where $\mu$ and $\nu$ are compactly supported measures on $\mathbb{R}$, together with connections with some operator theory questions, are presented in $[\mathrm{BF}]$. Most of these results (including the results of this paper) form part of the second author's Ph.D. dissertation [F] written under the direction of the first author.

\section{THE CLOSURE OF THE GRAPH OF A DIFFERENTIATION}

Let $E$ be a compact subset of the real line $\mathbb{R}$, and let $C(E)$ denote the Banach space of continuous functions on $E$ with the uniform norm. For convenience without loss of generality we assume that $E$ is contained in the unit interval $[0,1]$. Consider the densely defined operator $D: C(E) \rightarrow C(E)$ given by $D p=p^{\prime}$ for all polynomials $p$. Let $\mathscr{G}$ represent the graph of $D$, that is

$$
\mathscr{G}=\left\{\left(p, p^{\prime}\right): p \text { a polynomial }\right\} .
$$

We wish to characterize the closure of $\mathscr{G}, \mathscr{G}^{-}$in the product topology of uniform convergence in each component.

The analysis relies on the topological properties of $E$. Denote by int $E$ the interior of $E$. Write int $E$ as a countable disjoint union of intervals int $E=$ $\dot{\bigcup}_{i}\left(a_{i}, b_{i}\right)$. Let $\widehat{E}$ be given by $\widehat{E}=\dot{\bigcup}_{i}\left[a_{i}, b_{i}\right]$. Notice first that the expression for $\widehat{E}$ is also a disjoint union; indeed, if $\left[a_{i}, b_{i}\right] \cap\left[a_{j}, b_{j}\right] \neq \varnothing$ where $a_{i}<a_{j}$, then $\left(a_{i}, b_{j}\right) \subset$ int $E$, a contradiction. Secondly $E \backslash \widehat{E}$ contains no intervals since it is a subset of the nowhere dense set $E \backslash($ int $E)$. Now let $\hat{\mathscr{G}}$ denote the closed submanifold of $C(E) \times C(E)$ defined by

$$
\widehat{\mathscr{G}}=\left\{(h, k) \in C(E) \times C(E): h \text { is } A C(\widehat{E}) \text { and } k\left|\widehat{E}=h^{\prime}\right| \widehat{E}\right\} .
$$

Our result is that $\mathscr{G}^{-}=\widehat{\mathscr{G}}$. We begin by proving $\mathscr{G}^{-} \subset \widehat{\mathscr{G}}$, which is straightforward. The other inclusion relies on a characterization of the annihilator of $\mathscr{G}$, $\mathscr{G}^{\perp}$, where the duality relationship is that of $C(E)$ with $M(E)$, the Banach space of all regular Borel measures supported in $E$.

Theorem 2.2. $\mathscr{G}-\subseteq \widehat{\mathscr{G}}$.

Proof. Let $(h, k) \in \mathscr{G}^{-}$. Let $\left\{p_{n}\right\}$ be a sequence of polynomials such that $p_{n} \rightarrow h$ and $p_{n}^{\prime} \rightarrow k$ uniformly on $E$. If $\widehat{E}=\dot{\bigcup}_{i}\left[a_{i}, b_{i}\right]$, we need to show for each $i, h$ is $A C\left(\left[a_{i}, b_{i}\right]\right)$ and $\left.h^{\prime}\right|_{\left[a_{i}, b_{i}\right]}=k$. Fix one such $i$. We have, for all $x, y \in\left[a_{i}, b_{i}\right]$,

$$
p_{n}(x)-p_{n}(y)=\int_{[y, x]} p_{n}^{\prime}(t) d t .
$$

Furthermore for each $x, y \in\left[a_{i}, b_{i}\right], p_{n}(x)-p_{n}(y) \rightarrow h(x)-h(y)$.

Finally, since $p_{n}^{\prime} \rightarrow k$ uniformly, we have

$$
\lim _{n \rightarrow \infty} \int_{[y, x]} p_{n}^{\prime}(t) d t=\int_{[y, x]} k(t) d t
$$


for all $x, y \in\left[a_{i}, b_{i}\right]$. The theorem then follows by taking the limit as $n$ goes to infinity in (2.2.1).

The proof of the other inclusion involves establishing some lemmas which characterize $\mathscr{G}^{\perp}$ and localizes our concerns to each $\left[a_{i}, b_{i}\right]$.

Lemma 2.3. For two regular Borel measures $m_{1}$ and $m_{2}$ supported on $E$, $\left(m_{1}, m_{2}\right) \in \mathscr{G}^{\perp}$ if and only if

$$
\begin{gathered}
\int_{[0,1]} d m_{1}=0 \\
\text { the measure }\left[\int_{(x, 1]} d m_{1}(t)\right] d x \text { is supported in } E,
\end{gathered}
$$

and

$$
d m_{2}(x)=-\left[\int_{(x, 1]} d m_{1}(t)\right] d x .
$$

Proof. We first note that (2.3.3) states that the Radon-Nikodym derivative of $m_{2}$ with respect to $m$ (Lebesgue measure) is $-\left[\int_{(x, 1]} d m_{1}(t)\right]$. That is

$$
d m_{2} / d m=-\left[\int_{(x, 1]} d m(t)\right] .
$$

Now suppose $\left(m_{1}, m_{2}\right) \in \mathscr{G}^{\perp}$. Then for all polynomials $p$,

$$
\begin{aligned}
0 & =\int_{[0,1]} p(x) d m_{1}(x)+\int_{[0,1]} p^{\prime}(x) d m_{2}(x) \\
& =p(0) \int_{[0,1]} d m_{1}(x)+\int_{[0,1]}\left(\int_{[0, x]} p^{\prime}(t) d t\right) d m_{1}(x)+\int_{[0,1]} p^{\prime}(x) d m_{2}(x) \\
& =p(0) \int_{[0,1]} d m_{1}(t)+\int_{[0,1]} p^{\prime}(x)\left(\int_{(x, 1]} d m_{1}(t)\right) d x+\int_{[0,1]} p^{\prime}(x) d m_{2}(x)
\end{aligned}
$$

Letting $p \equiv 1$ in (2.3.4), we get that $m_{1}$ must satisfy (2.3.1). Next, by considering polynomials $p^{\prime}$ with $p(0)=0$ (a dense set in $C([0,1])$ by the StoneWeierstrass theorem), it follows from (2.3.4) that $m_{1}$ and $m_{2}$ must satisfy (2.3.2) and (2.3.3).

To prove the other direction simply reverse the argument.

The key to the proof of the general result is that elements in $\mathscr{G}^{\perp}$ must be zero off $\widehat{E}$. This is established in the following.

Lemma 2.4. If $\left(m_{1}, m_{2}\right) \in \mathscr{G}^{\perp}$, then $m_{1}$ has no point masses in $E \backslash \widehat{E}$.

Proof. As noted above, $E \backslash \widehat{E}$ contains no intervals. Thus any open interval must intersect the complement of $E \backslash \widehat{E}$. Let $x_{0} \in E \backslash \widehat{E}$. By (2.3.2), for $m-$ a.e. $x \in[0,1] \backslash E$

$$
m_{1}((x, 1])=\int_{(x, 1]} d m_{1}(t)=0
$$


Thus, by additivity, $m_{1}((x, y])=0$ for $m$ - a.e. $x, y$ in $[0,1] \backslash E$. Now choose a sequence of positive numbers $\left\{\xi_{n}\right\}$ so that $\xi_{n} \searrow 0$. Let $I_{n}=\left(x_{0}-\right.$ $\left.\xi_{n}, x_{0}+\xi_{n}\right)$. We wish to show that, for each $n$, there are points $x_{n}$ and $y_{n}$ belonging to $I_{n}$, with $x_{n}<x_{0}<y_{n}$, so that $m_{1}\left(\left(x_{n}, y_{n}\right]\right)=0$. To do this, from (2.4.1), it suffices to show that each $I_{n}$ intersects the complement of $E$ in a set of positive Lebesgue measure on each side of $x_{0}$. We first show that $I_{n}$ must intersect the complement of $E$ on each side of $x_{0}$. Indeed, if, say, $I_{n}$ did not intersect $[0,1] \backslash E$ to the left of $x_{0}$, then it would follow that $\left(x_{0}-\xi_{n}, x_{0}\right] \subset E$. But this would contradict $x_{0} \notin \widehat{E}$. Next, note that both $[0,1] \backslash E$ and $I_{n}$ are open. Thus their nonempty intersection, which, from above, contains an open interval on each side of $x_{0}$, must have positive Lebesgue measure. Thus we have shown that there are sequences $\left\{x_{n}\right\},\left\{y_{n}\right\}$ such that $\bigcap_{n}\left(x_{n}, y_{n}\right]=\left\{x_{0}\right\}$ and $m_{1}\left(\left(x_{n}, y_{n}\right]\right)=0$ for each $n$. Finally, since $m_{1}$ is regular, we have

$$
\lim _{n \rightarrow \infty} m_{1}\left(\left(x_{n}, y_{n}\right]\right)=m_{1}\left(\left\{x_{0}\right\}\right)
$$

and the lemma follows.

Lemma 2.5. Let $A=\left\{x \in E \backslash \widehat{E}: \int_{(x, 1]} d m_{1}(t)=0\right\}$. Then $m_{1}(A)=0$.

Proof. We use in our arguments the total variation of $m_{1},\left|m_{1}\right|$, which is regular if $m_{1}$ is regular. So, without loss of generality, we may assume $A$ is closed. Let $\varepsilon>0$ be given and choose an open set $U \supset A$ so that $\left|m_{1}\right|(U \backslash A)<$ $\varepsilon$. Write $U$ as a disjoint union of open intervals: $U=\dot{\bigcup}_{n}\left(\alpha_{n}, \beta_{n}\right)$. We construct a new open set $U_{1} \supset A$ as follows. Delete each interval $\left(\alpha_{n}, \beta_{n}\right)$ for which $\left(\alpha_{n}, \beta_{n}\right) \cap A=\varnothing$ from the collection $\left\{\left(\alpha_{n}, \beta_{n}\right)\right\}$. Let

$$
\alpha_{n}^{\prime}=\inf \left\{x: x \in\left(\alpha_{n}, \beta_{n}\right) \cap A\right\}, \quad \beta_{n}^{\prime}=\sup \left\{x: x \in\left(\alpha_{n}, \beta_{n}\right) \cap A\right\} .
$$

Then, for each $n$ not deleted, let

$$
U_{1}=\bigcup_{n}\left(\alpha_{n}^{\prime}, \beta_{n}^{\prime}\right) \text {. }
$$

Then $A \subset U_{1}$, modulo the endpoints $\alpha_{n}^{\prime}$ and $\beta_{n}^{\prime}$ which are in $A$, since $A$ is closed. But the collection of all endpoints is a countable set in $E \backslash \widehat{E}$, and, by Lemma 2.4, has $m_{1}$-measure zero. Furthermore,

$$
m_{1}\left(\left(\alpha_{n}^{\prime}, \beta_{n}^{\prime}\right)\right)=\int_{\left(\alpha_{n}^{\prime}, 1\right]} d m_{1}(t)-\int_{\left(\beta_{n}^{\prime}, 1\right]} d m_{1}(t)=0
$$

by assumption. Thus $m_{1}\left(U_{1}\right)=0$. Finally, then

$$
\begin{aligned}
\left|m_{1}(A)\right| & =\left|m_{1}\left(U_{1}\right)-m_{1}(A)\right|=\left|m_{1}\left(U_{1} \backslash A\right)\right| \\
& \leq\left|m_{1}\right|\left(U_{1} \backslash A\right) \leq\left|m_{1}\right|(U \backslash A)<\varepsilon .
\end{aligned}
$$

So by arbitrariness of $\varepsilon$, Lemma 2.5 follows.

Lemma 2.6. For $\left(m_{1}, m_{2}\right) \in \mathscr{G}^{\perp},\left|m_{1}\right|(E \backslash \widehat{E})=0$.

Proof. Suppose there is an $A \subset E \backslash \widehat{E}$ with $m_{1}(A) \neq 0$. Then by Lemma 2.4, there exists $x_{0} \in E \backslash \widehat{E}$ so that

$$
\int_{\left(x_{0}, 1\right]} d m_{1}(t) \neq 0 .
$$


But, since $m_{1}$ has no point masses in $E \backslash \widehat{E}$, the function $\int_{(x, 1]} d m_{1}(x)$ is continuous on $E \backslash \widehat{E}$.

Thus there is an interval $I$ containing $x_{0}$ and $\xi>0$, so that

$$
\left|\int_{(x, 1]} d m_{1}(t)\right|>\xi
$$

for all $x \in I \cap(E \backslash \widehat{E})$. Since $I$ must intersect $[0,1] \backslash E$ and $m_{1}$ is supported on $E$, this contradicts (2.3.2).

Corollary 2.7. For $\left(m_{1}, m_{2}\right) \in \mathscr{G}^{\perp},\left|m_{2}\right|(E \backslash \widehat{E})=0$.

Proof. Let $B \subset E \backslash \widehat{E}$. Then, if $A$ is defined as in Lemma 2.5,

$$
B=(B \cap A) \dot{\cup}(B \cap[(E \backslash \widehat{E}) \backslash A])=B_{1} \dot{\cup} B_{2} .
$$

Now, by $(2.3 .3)$, for any set $C$

$$
m_{2}(C)=\int_{C} d m_{2}(t)=-\int_{C} \int_{(x, 1]} d m_{1}(t) d x .
$$

So $m_{2}\left(B_{1}\right)=0$ by definition of $A$. So suppose $m_{2}\left(B_{2}\right) \neq 0$. Then there must be an $x_{0} \in E \backslash \widehat{E}$ so that $\int_{\left(x_{0}, 1\right]} d m_{1}(t) \neq 0$. But this leads to a contradiction as in the proof of Lemma 2.6.

Recall that $\left[a_{i}, b_{i}\right](i=1,2, \ldots)$ are the maximal intervals in $\widehat{E}$.

Lemma 2.8. For each $i$,

Proof. By regularity of $m$, for $x_{n} \nearrow a_{i}$

$$
0=\int_{\left[a_{i}, 1\right]} d m_{1}(t)=\int_{\left(b_{i}, 1\right]} d m_{1}(t) .
$$

$$
\lim _{n \rightarrow \infty} m_{1}\left(\left(x_{n}, 1\right]\right)=m_{1}\left(\left[a_{i}, 1\right]\right) \text {. }
$$

Let $I$ be an open interval containing $a_{i}$. Then $I$ must intersect $[0,1] \backslash E$, an open set of positive Lebesgue measure. But since (2.3.2) states $\int_{(x, 1]} d m(t)=0$ for $m$ - a.e. $x \in[0,1] \backslash E$, there is a sequence $\left\{x_{n}\right\}$ converging up to $a_{i}$ so that, for each $n$,

$$
m_{1}\left(\left(x_{n}, 1\right]\right)=\int_{\left(x_{n}, 1\right]} d m_{1}(t)=0 .
$$

Using this sequence in (2.8.1), we get $\int_{[a, 1]} d m(t)=0$. A similar argument shows that $\int_{\left(b_{i}, 1\right]} d m_{1}(t)=0$.

Theorem 2.9. $\hat{\mathscr{G}} \subseteq \mathscr{G}$ -

Proof. Let $(h, k) \in \hat{\mathscr{G}}$. We show $(h, k) \in \mathscr{G}^{-}$by showing

$$
\int_{[0,1]} h(x) d m_{1}(x)+\int_{[0,1]} k(x) d m_{2}(x)=0
$$

for all $\left(m_{1}, m_{2}\right) \in \mathscr{G}^{\perp}$. But, using Lemma 2.6 and Corollary 2.7, it suffices to show for each $i$ and for each $\left(m_{1}, m_{2}\right) \in \mathscr{G}^{\perp}$

$$
\int_{\left[a_{i}, b_{i}\right]} h(x) d m_{1}(x)+\int_{\left[a_{i}, b_{i}\right]} k(x) d m_{2}(x)=0 .
$$


So fix $i$, and let $\left(m_{1}, m_{2}\right) \in \mathscr{G}^{\perp}$. Using (2.3.3) we have

$$
\begin{aligned}
\int_{\left[a_{i}, b_{i}\right]} k(x) d m_{2}(x)= & -\int_{\left[a_{i}, b_{i}\right]} k(x)\left(\int_{(x, 1]} d m_{1}(t)\right) d x \\
= & -\int_{\left[a_{i}, b_{i}\right]}\left(\int_{\left[a_{i}, x\right]} k(t) d t\right) d m_{1}(x) \\
& -\int_{\left(b_{i}, 1\right]}\left(\int_{\left[a_{i}, b_{i}\right]} k(t) d t\right) d m_{1}(x)
\end{aligned}
$$

by interchanging the order of integration. But, by assumption, $h(y)-h(x)=$ $\int_{[x, y]} k(t) d t$ for all $x, y \in\left[a_{i}, b_{i}\right]$. Thus,

$$
\begin{aligned}
\int_{\left[a_{i}, b_{i}\right]} k(x) d m_{2}(x)= & -\int_{\left[a_{i}, b_{i}\right]} h(x) d m_{1}(x)+h\left(a_{i}\right) \int_{\left[a_{i}, b_{i}\right]} d m_{1}(x) \\
& -h\left(b_{i}\right) \int_{\left(b_{i}, 1\right]} d m_{1}(x)+h\left(a_{i}\right) \int_{\left(b_{i}, 1\right]} d m_{1}(x) \\
= & -\int_{\left[a_{i}, b_{i}\right]} h(x) d m_{1}(x)+h\left(a_{i}\right) \int_{\left[a_{i}, 1\right]} d m_{1}(x) \\
& -h\left(b_{i}\right) \int_{\left(b_{i}, 1\right]} d m_{1}(x) .
\end{aligned}
$$

So

by Lemma 2.8 .

$$
\begin{aligned}
& \int_{\left[a_{i}, b_{i}\right]} h(x) d m_{1}(x)+\int_{\left[a_{i}, b_{i}\right]} k(x) d m_{2}(x) \\
& =h\left(a_{i}\right) \int_{\left[a_{i}, 1\right]} d m_{1}(x)-h\left(b_{i}\right) \int_{\left(b_{i}, 1\right]} d m_{1}(x)=0
\end{aligned}
$$

\section{THE CLOSABILITY OF DIFFERENTIATION}

AND SIMULTANEOUS APPROXIMATIONS

We conclude by describing when $D$ is closable and when $\mathscr{G}^{-}$is all of $C(E) \times$ $C(E)$. The latter provides the solution to the approximation problem initially addressed.

Theorem 3.1. $D$ is closable if and only if $E=(\widehat{E})^{-}$(that is, $E$ is the closure of its interior).

Proof. First suppose $(\widehat{E})^{-}=E$. Let $(0 \oplus g) \in \mathscr{G}^{-}$. Then, by Theorem 2.2, $g \equiv 0$ on $\widehat{E}$. Thus, by continuity, $g \equiv 0$ on $(\widehat{E})^{-}$.

Conversely, suppose $(\widehat{E})^{-} \neq E$. Let $x_{0} \in E \backslash(\widehat{E})^{-}$. By Urysohn's Lemma, there is a function $g$, continuous on $E$, so that $g\left(x_{0}\right)=1$ and $g(x)=0$ for all $x \in(\widehat{E})^{-}$. Now $(0, g) \in \widehat{\mathscr{G}} \subset \mathscr{G}^{-}$, by Theorem 2.9 , but is nonzero. Thus $D$ is not closable.

Theorem 3.2. $\mathscr{G}^{-}=C(E) \times C(E)$ if and only if $\widehat{E}=\varnothing$ (that is, $E$ has empty interior).

Proof. If $\widehat{E} \neq \varnothing$, we have for $h(x) \equiv 1$ on $E,(h \oplus h)$ is in $C(E) \oplus C(E)$ but not in $\mathscr{G}^{-}$. Conversely, if $\widehat{E}=\varnothing$, then there are no requirements on an element of $C(E) \times C(E)$ to be in $\hat{\mathscr{G}}_{C} \mathscr{G}^{-}$. Thus $\mathscr{G}^{-}=C(E) \times C(E)$. 
A reformulation of Theorem 3.2 is as follows.

Corollary 3.3. If $E$ is a compact subset of the real line and $f$ and $g$ are arbitrary continuous on $E$, then there is a sequence of polynomials $\left\{p_{n}\right\}$ so that $p_{n} \rightarrow f$ and $p_{n}^{\prime} \rightarrow g$ uniformly on $E$ if and only if $E$ has empty interior.

\section{REFERENCES}

[B1] E. Bishop, Approximation by a polynomial and its derivatives on certain closed sets, Proc. Amer. Math. Soc. 9 (1958), 946-953.

[B2] - Simultaneous approximation by a polynomial and its derivatives, Proc. Amer. Math. Soc. 10 (1959), 741-743.

[BF] J. A. Ball and T. R. Fanney, Closability of differential operators and real subjordan operators, Topics in Operator Theory: Ernst D. Hellinger Memorial Volume, (L. de Branges, I. Gohberg, and J. Rounyak, eds.), Operator Theory: Advances and Applications, vol. 48, Birkhäuser-Verlag, Basel, 1990, pp. 93-156.

[CV] C. O. Christenson and W. L. Voxman, Aspects of topology, Marcel Dekker, New York, 1977.

[F] T. R. Fanney, Closability of differential operators and subjordan operators, Ph.D. dissertation, Virginia Polytechnic Institute and State University, 1989.

[FR] U. Fixman and L. A. Rubel, Limits of sequences of rational functions and their derivatives, Houston J. Math. 5 (1979), 75-87.

[R] W. Rudin, Functional analysis, McGraw-Hill, New York, 1973.

Department of Mathematics, Virginia Polytechnic Institute and State University, BLACKSBURG, VIRGINIA 24061

Department of Mathematics, Virginia Wesleyan College, Wesleyan College, NorFOLK, VIRGINIA 23502 